\title{
Novel Implementation of the Artificial Immune System Memory
}

\author{
Hossam Meshref \\ Associate Professor, Computer Science Department \\ College of Computers and Information Technology \\ Taif University, Kingdom of Saudi Arabia
}

\begin{abstract}
During the past decade, there were many approaches that made use of the concepts of artificial immune system. By reviewing the literature, it was found that the research on the artificial immune memory design was sparse and only a few researchers attempted to embark upon issues related to it. This proposed research introduces a novel model that serves as an artificial memory for the artificial immune system. The proposed research design approaches this goal by modeling the primary immune memory response using an idiotopic artificial immune network. As well, the secondary immune memory response is modeled by using a hetero-associative memory network. The two models were tested and the results showed considerable resemblance with the primary and secondary immune memory system behavior. The testing results showed that the developed memory models achieved $100 \%$ recognition rates for the tested patterns. Even after adding noise to the input data, the overall memory model showed acceptable recognition rate of $87.5 \%$ for $25 \%$ noise, and $68.7 \%$ recognition rate for $50 \%$ noise. Therefore, the designed primary and secondary artificial immune memory model achieve significant potential in representing the behavior of the original immune system memory.
\end{abstract}

\section{Keywords}

Artificial Immune Systems, Artificial Immune Memory, Idiotopic Artificial Immune Network, Hetero-Associative Networks

\section{INTRODUCTION}

The artificial immune system is modeled after the biological immune system, which has many features. For example, it adapts to changes in the environment, it has hierarchical organization and its control is distributed. The biological immune system consists mainly of lymphocytes that have two major types, T-cells and B-cells. B-cells are responsible for humoral immunity that secretes antibodies, while T-cells are responsible for cell mediated immunity. Each B-Cell has a unique structure that produces suitable antibodies in response to invading antigens. To produce such response the B-cell has to undergo proliferation and differentiation. Similarly, when the receptors of a T-cell bind to an antigen, it undergoes proliferation. In the early stages of the primary immune response, affinity is low, however clones mutate during the somatic hypermutation process, see figure 1 . Eventually, the activated B-cells will mature into a pool of antibody-secreting plasma cells and a pool of memory cells $[1,2]$.

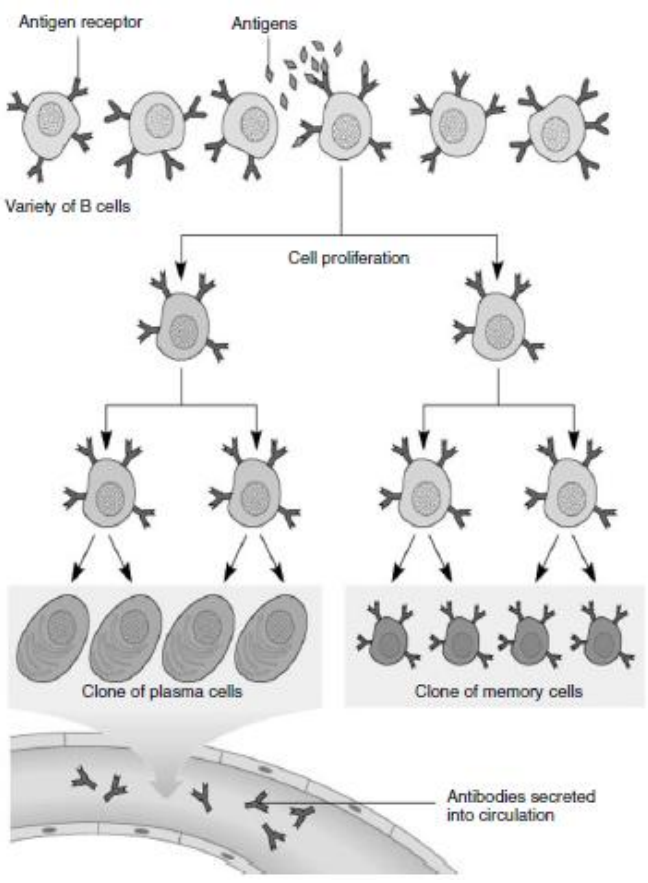

Fig. 1. Generation of Memory Cells in the Artificial Immune System [1]

There were many approaches that made use of the concepts of artificial immune system. Many researchers have tried to investigate it to understand its capabilities and limitations. Take applications on the field of robotics for example. Dasgupta and Nino, in their book, surveyed robotics research inspired by Artificial Immune Networks up to 2009 [3]. Among research done on autonomous robots there were a few robots that utilized a mechanism called Immunoid to collect various amount of garbage from a constantly changing environment [4]. Singh and Thayer used a computer simulation of 2750 mobile robots trying to clear 7500 mines from a mine field using a response pattern based on the immune system [5]. Their research used a general suppression control framework that is based on suppression mechanism of immune cells. There are many applications that benefit from the immune system network features like self non-self discrimination, and specificity [6,7]. The main idea behind their research was having a network that adapts its behavior by adjusting the concentrations of its nodes, Abs in each BCell, in a way that fulfills the overall objective function of the network. Each node in these network represents a robot that has certain set of behaviors, and collectively the network adapts robots behaviors as they collaborate to achieve an overall common goal. Once more, the aforementioned designed systems show the Ability of the artificial immune network to learn using stimulation and suppression among 
network components behaviors while learning to achieve a certain goal.

By reviewing the literature, it was found that the research on the artificial immune memory was sparse and only a few researchers attempted to embark upon issues related to the immune memory. For example, Chung-Ming and C.R. OU proposed a dynamic system that explains the formation of memory states and how that leads to the stabilization of the overall immune network. However, their work was focused on the biological aspect of the memory and not on the artificial depiction of the immune memory within the network $[8,9]$.

Alexandrino et al created a hybrid immune algorithm to maintain high affinity antibody to be stored in the memory during an evolutionary learning process [10]. Their goal was to cluster the data based on the algorithms of Adaptive Resonance Theory. They modified their algorithm with clonal selection strategies in order to come up with the best data set to be stored in the immune memory. A similar work by Yap and Tiong focused on the pre-processing of data, antibodies with high affinity, before storing it the memory [11]. They focused of how to improve the regular search algorithms that used to utilize clonal selection, swarm intelligence, and genetic algorithms by designing a hybrid model.

Most of the aforementioned research work approaches focus on improving the data to be stored in the artificial immune memory. Although the aforementioned research work showed promising results in their research topic, they did not focus on the memory design structure, which is expected to improve the overall artificial immune network performance. Finding a robust model for the memory structure could improve the primary and the secondary response when the network encounters new patterns. That in turn, from the general artificial immune network point of view, would increase the precision of memory response dynamics within the network. The purpose of this research is to investigate a possible artificial immune memory design model that could behave as closely as possible to the biological immune system memory.

Section two sheds light on the biological primary and secondary immune memory responses. Section three describes the rod bearing problem that is used as an autonomous robotics model for simulation purposes. Section four explains the implementation of the primary immune memory response network based on the ideology of an idiotopic artificial immune network. Section five, on the other hand, focuses on the secondary immune response and its implementation using a hetero-associative networks. Research results are discussed in section six, followed by the conclusion along with the future work research directions for interested researchers in section seven. The designed memory model is expected to be useful in similar applications that focus on improving the learning process within the artificial immune network.

\section{THE IMMUNE SYSTEM MEMORY}

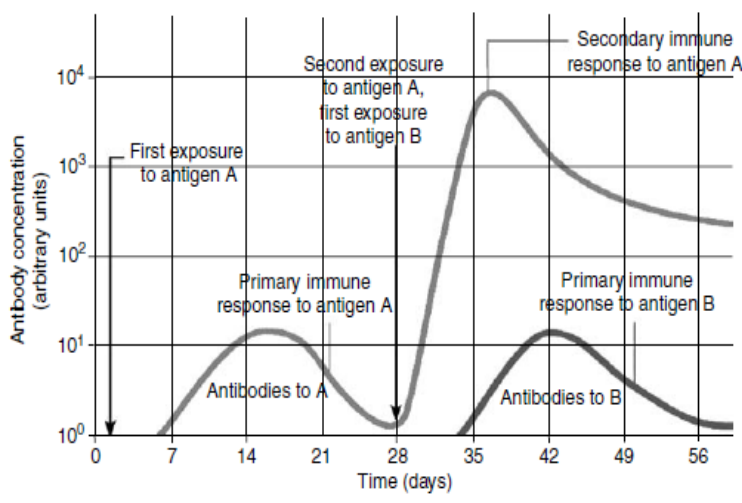

Fig. 2. The Production of Antibodies during the Primary Response and Secondary Response [1]

Figure 2 shows that, the biological immune network takes some time to come up with a set of antibodies that can recognize an antigen ' $A$ '. Once determined, it is cloned, and later on stored in the immune system memory for future antigen encounters. This process takes place during the primary immune response, which is relatively slow compared to the secondary immune response. That behavior resembles the behavior of the idiotopic artificial immune network. During the secondary immune response, the memory cells help in accelerating the interaction with the antigen. This goal is fulfilled by recalling the corresponding antibody, stored in the memory, which had previously recognized that antigen. If recognized with memory help, that will result in an increase in the number of the antibodies needed to overcome the recognized antigen ' $A$ '. That latter behavior resembles the behavior of the hetero-associative memory model. Based on the aforementioned resemblances, it is anticipated that investigating the idiotopic artificial immune network and the associative memory network models will improve the artificial memory performance during the primary and the secondary responses.

\section{THE ROD BEARING PROBLEM}

The Rod-Bearing problem is a challenge in which two autonomous mobile robots, robot-team, working in a hazardous environment, are trying to carry a rod together in order to deliver it into a specified target area. The robot-team has to adapt their behavior selection in such a way that guarantees the successful delivery of the rod into the designated target area [12]. This simple experiment was used in this research for simulation purposes to help in investigating the designed artificial memory models. It is imperative to discuss the robot kinematics of a differential wheel mobile robot simulated in this research. Basically, varying the velocities of the two wheels of each robot will lead to varying the trajectories that the robot takes. The instantaneous centre of curvature (ICC) must be the same for both wheels. Based on that assumption, the following equations could be deduced:

$$
\begin{aligned}
& V_{r}=\omega(R+l / 2) \\
& V_{l}=\omega(R-l / 2)
\end{aligned}
$$

Where $l$ is the distance between the centers of the two wheels, $\mathrm{V}_{\mathrm{r}}, \mathrm{V}_{1}$ are the right and left wheel velocities. $\mathrm{R}$, on the other hand, is the distance from the ICC to the midpoint between the two wheels. Based on these assumptions, the following 
equations could be deduced:

$$
\begin{gathered}
R=\frac{l}{2} \times \frac{V_{l}+V_{r}}{V_{r}-V_{l}} \quad(3) \\
\omega=\frac{V_{r}-V_{l}}{l} \quad(4) \\
I C C=[x-R \sin \theta, y+R \cos [(\theta)]
\end{gathered}
$$

In the beginning, each robot is at some position $(\mathrm{x}, \mathrm{y})$, moving in a certain direction making an angle $\theta$ with the $X$ axis. By manipulating the control parameters $V_{1}$ and $V_{r}$, the robot moves to different positions and orientations. By knowing velocities $\mathrm{V}_{\mathrm{l}}, \mathrm{V}_{\mathrm{r}}$ and the ICC location, new location of robot-team $\left(x^{`}, y^{`}\right)$ can be identified using equation 6 :

$$
\left[\begin{array}{l}
x^{\prime} \\
y^{\prime} \\
\theta^{\prime}
\end{array}\right]=\left[\begin{array}{ccc}
\cos (\omega \delta t) & -\sin (\omega \delta t) & 0 \\
\sin (\omega \delta t) & \cos (\omega \delta t) & 0 \\
0 & 0 & 1
\end{array}\right]\left[\begin{array}{c}
x-I C C_{x} \\
y-I C C_{y} \\
\theta
\end{array}\right]+\left[\begin{array}{c}
I C C_{x} \\
I C C_{y} \\
\omega \delta t
\end{array}\right]
$$

\section{MODELING THE PRIMARY IMMUNE SYSTEM MEMORY RESPONSE}

The design of the idiotopic artificial immune network, used to simulate the primary immune response, is based upon Jerne's biological approach [13, 14]. In his approach, Jerne discovered that the immune system has Idiotopic networks that use stimulation and suppression among its elements to achieve immunity against antigens. The part of an antigen that can be recognized by antibodies is called epitope. As a result of this stimulation, the B-cells start to produce Abs. On the other hand, the part of the antibody that can recognize epitopes of antigens is called paratope. However, part of an antibody, called idiotope, could be regarded as antigen by other antibodies in the idiotopic network.

Figure 3 shows the direct mapping used in this research design between robot sensory information and the corresponding effectors. It mimics the innate responses and reflexes in the artificial immune system. That means the precomputed reflexes, robot response actions, are guaranteed to be fast for most of the environment changes that surround the robot. For action selection, the IAIN controller may select one output action, using arbitration, but may not combine more than one action to achieve the desired result.

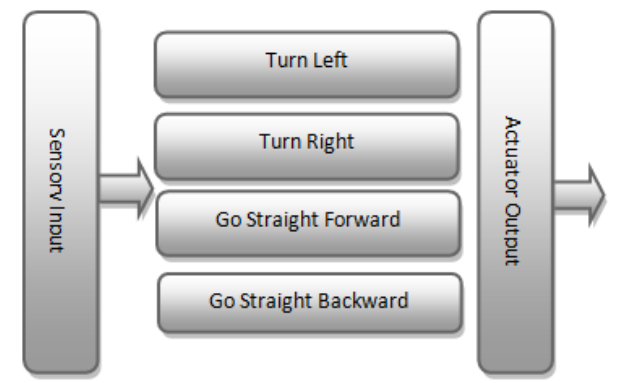

Fig. 3. Parallel Concurrent Task Achieving Module Used within the Idiotopic Immune Network (IAIN) Controller

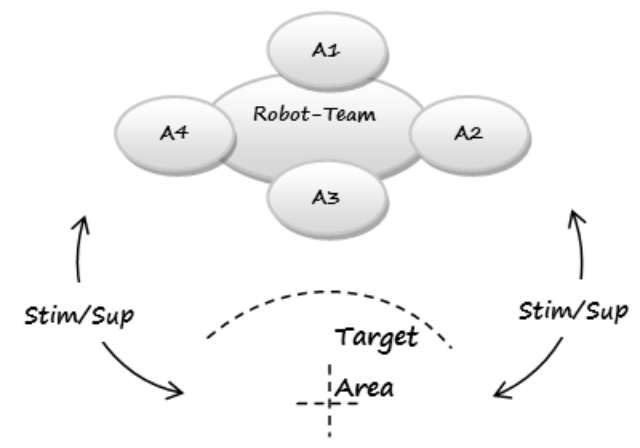

Fig. 4. IAIN During Ag Identification Phase: Primary Immune Response

Figure 4 illustrates the proposed IAIN network structure, which consists of robot-team, B-cell, with a few set of possible behaviors: $A_{i}$ 's. The distance between the robot-team and the target area is D. As long as robot-team is out of the target area, there will always be stimulation from Ag to the $\mathrm{Ab}$. From the artificial immune system point of view, D is the measure of affinity between an Ag and Ab, and it could be calculated using the Euclidean distance equation. However, action arbitration is done based on the interaction, stimulation (stim) and suppression (sup), between the elements of the idiotopic artificial immune network. Table 1, in the next page, shows the possible robot-team behaviors and their abbreviations during rod-bearing problem simulation.

Table I. Possible Behaviors and their Abbreviations

\begin{tabular}{ll}
\hline Action & Behavior \\
\hline$A_{1}$ & Turn left with angle $\theta$ \\
$A_{2}$ & Turn right with angle $\theta$ \\
$A_{3}$ & Go straight forward \\
$A_{4}$ & Go straight backward \\
\hline
\end{tabular}

During simulation, each robot has a state that is considered to be a B-cell and its behaviors are considered to be the antibodies. The main objective of the robot-team is to deliver the rod to the target area, within $R \tau$ distance range, and as a result any behavior that leads otherwise needs to be avoided as much as possible. The Idiotopic artificial immune network is expected to use its stimulation and suppression mechanisms to stimulate robot-team movements that help reaching the target, and at the same time suppresses those movements who lead away from it. In general, researchers claimed that the interaction within the Idiotopic immune network could be governed by the following equation:

$\frac{d c_{i}}{d t}=\sum_{j=1}^{N} m_{j, i} c_{i} c_{j}-k_{3} \sum_{k=1}^{N} m_{i, k} c_{i} c_{k}+k_{4} m_{i} c_{i}-k_{5} c_{i}$

Where the first term, from the left, represents the total stimulation between different B-cells in he idiotopic network, while the second term represents the total suppression. The third term represents an $\mathrm{Ab}$ stimulation received by an Ag. As for the fourth term it represents the mortality of an $\mathrm{Ab}$ $[15,16,17,18]$. In this proposed research, both robots act together, which means that niether stimulation nor suppression is allowed to happen between them. However, it is only allowed between an $\mathrm{Ab}$ and an $\mathrm{Ag}$ within the same network. Therefore, equation 7 could be simplified to be equation 8. Another step was needed to garuantee the stability of concentraion values by deploying a squashing function, see equation 9. 


$$
\begin{aligned}
\frac{d c_{i}}{d t} & =k_{4} m_{i} c_{i}-k_{5} c_{i} \\
c_{i} & =\frac{1}{1+e^{\left(0.5-c_{i}\right)}}
\end{aligned}
$$

The overall dynamics within the designed IAIN controller

\begin{tabular}{|c|c|}
\hline \multicolumn{2}{|c|}{ Primary Immune Memory Response } \\
\hline 1: & Do While $\left(D>R_{\tau}\right)$ \\
\hline 2: & Check the Immune Memory for a response \\
\hline 3: & If there is a suitable response, then \\
\hline 4: & Execute behavior \\
\hline 5: & Break \\
\hline & Else \\
\hline 6: & Check behaviors' concentrations \\
\hline 7: & Select behavior with highest concentration \\
\hline 8: & If all are the same, then \\
\hline & $\begin{array}{l}\text { Pick behavior from the available } \\
\text { behaviors' list }\end{array}$ \\
\hline 9: & Execute behavior \\
\hline 10: & Update concentrations \\
\hline 11: & Check behavior effect on objective function \\
\hline 12: & If the objective function is fulfilled, then \\
\hline 13: & Add behavior to memory \\
\hline 14: & EndIf \\
\hline 15: & Check the environment \\
\hline 16: & Check Robot-Team State \\
\hline 17: & EndWhile \\
\hline
\end{tabular}
could be explained by the algorithm shown below:

\section{MODELING THE SECONDARY IMMUNE SYSTEM MEMORY RESPONSE}

One of the most successful learning models in artificial neural networks is the Associative Memories. An associative memory is designed as a content-addressable structure. Its function is to map a set of input patterns into a set of output patterns. There are two types of associative memories: AutoAssociative and Hetero-Associative. The two network structures are different in the way that the input-output relations are modeled. The former retrieves the pattern that most closely resembles the input pattern. The latter retrieves a pattern that is associated with the input pattern $[19,20]$

There are two stages that are needed in the learning process in the designed associative memory: memorization and recall. During the memorization phase, the connection matrix, $\mathrm{W}_{\mathrm{k}}$, is formed by summing all the correlation matrices, where $\alpha$ is the proportionality constant:

$$
w=\alpha \sum_{k=1}^{p} w_{k}, p=4
$$

The correlation matrix $\mathrm{W}_{\mathrm{k}}$ is calculated using the associated pattern pairs $\left(\mathrm{X}_{\mathrm{k}}, \mathrm{Y}_{\mathrm{k}}\right)$, where:

$$
\left(w_{i j}\right)_{k}=\left(x_{i}\right)_{k}\left(y_{i}\right)_{k}
$$

During the recall phase, the net input is computed, which is the linear combination of the weighted input:

$$
y_{\text {net }}=\sum_{k}^{p} x_{i} w_{i}, p=4
$$

Then, using a bipolar threshold activation function, with threshold $\theta, Y_{\text {out }}$ is calculated:

$$
y_{\text {out }}= \begin{cases}+1, & y_{\text {net }} \geq \theta \\ -1, & y_{\text {net }}<\theta\end{cases}
$$

In this research, the associative network recognition ability was tested to retrieve the stored patterns as well as suggesting the closest match for the encountered antigen. Since binary input data was tested as well, therefore, a binary threshold function had to be used:

$$
y_{\text {out }}=\left\{\begin{aligned}
+1, & y_{\text {net }} \geq \theta \\
0, & y_{\text {net }}<\theta
\end{aligned}\right.
$$

In this research, as mentioned earlier, the hetero-associative memory network model is used to represent the secondary immune memory. Figure 5, depicts the designed network structure, where the network has two input vectors representing the environmental conditions: robot-team location and target location. The first input is a four element vector, which represents the status of the robot-team. As well, the second input is a four element vector, which represents the status of the target.

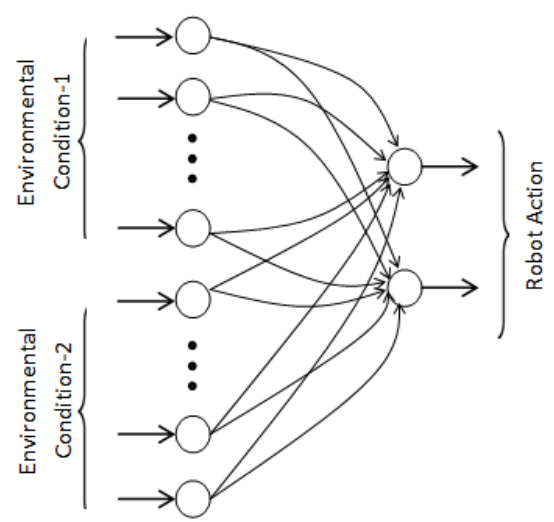

Fig. 5. The Designed Hetero-Associative Memory

\section{RESULTS AND DISCUSSION}

In this research, two simulations took place, the primary immune response simulation and the secondary immune response simulation. During the primary immune response simulation, the robot-team locations were decided based on the four cardinal directions: N, E, S, and W. The simulation arena was divided into four quadrants: Q1, Q2, Q3, and Q4. The robot-team behavior was controlled by the idiotopic artificial immune network. During the primary response simulation, the complete set of condition-action rules' associations covering the four quadrants were deducted, see figure 6 for a sample.
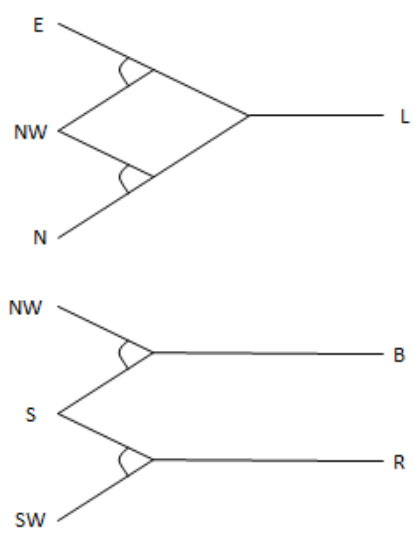

Fig. 6. Some Association Rules Generated during the Primary Immune Response 
During the primary immune response simulation, the robotteam distance to target was changing, while the robot-team was approaching the target area. On the other hand, during the secondary immune response simulation, the designed heteroassociative memory network came into play. The robot-team was able to find its path towards the target in steady steps, see figure 7 for distance comparison.

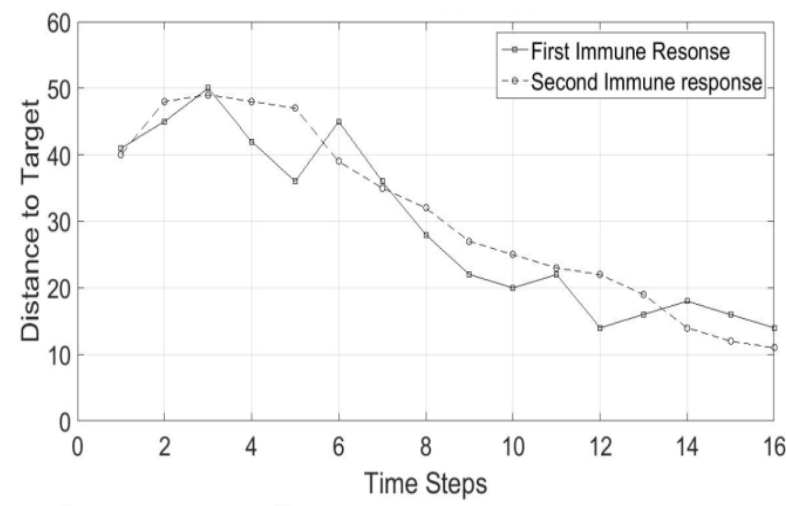

Fig. 7. Robot-Team Performance During Primary and Secondary Immune Responses

It is clear that during primary immune response, the robotteam distance was oscillating trying to minimize the distance to target. As rule associations were generated during simulation, the correct behavior was build gradually during learning causing such oscillations. However, during the secondary immune response, while using knowledge stored in the memory, the designed associative memory model guided the robot-team to proceed with a monotonically decreasing distance towards the target. The aforementioned simulations considerable resembles the behavior of the biological primary and secondary immune responses.

During the secondary immune response, the designed memory model was tested with binary and bipolar input vectors with and without noise. For binary input vectors with no noise in test vector, the success rate for hetero-associative network was $100 \%$. Next, with one-bit noise in the test vector, the success rate was $87.5 \%$. Finally, with two-bit noise in the test vector, the success rate was $50 \%$. Figure 8 , in the next page, shows the success ratio for binary input vectors with and without noise.

As well, for Bipolar input vectors, the model recognition ability was tested with 3 cases: With No noise in the test data, with one-bit noise, and with two-bit noise. For noise free input vectors, the success rate for the hetero associative network was $100 \%$. Next, the model was tested with one-bit noise in the test vector, and the success rate was $87.5 \%$. With two-bit noise, the success rate was $68.7 \%$. Figure 9, in the next page, shows the success ratio for bipolar input vectors with and without noise. Generally, it was noticed that the use of bipolar representation as a base during input preprocessing improves the designed memory model recognition ratio.

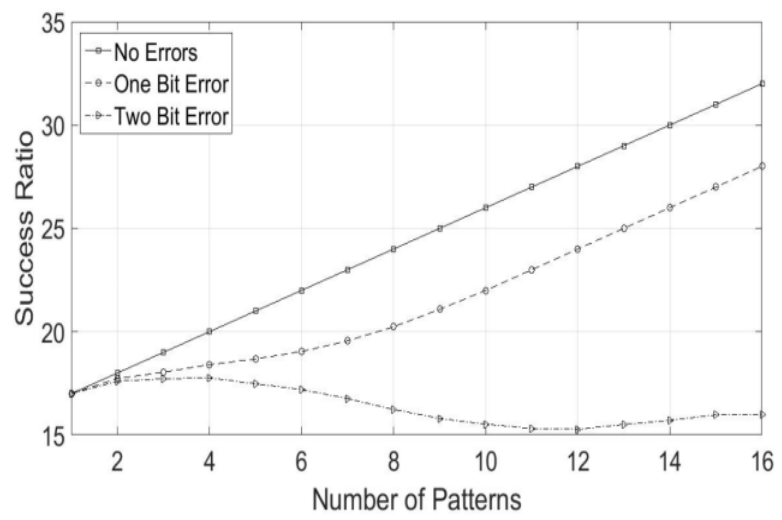

Fig. 8. Recognition Success Ratio for Binary Inputs

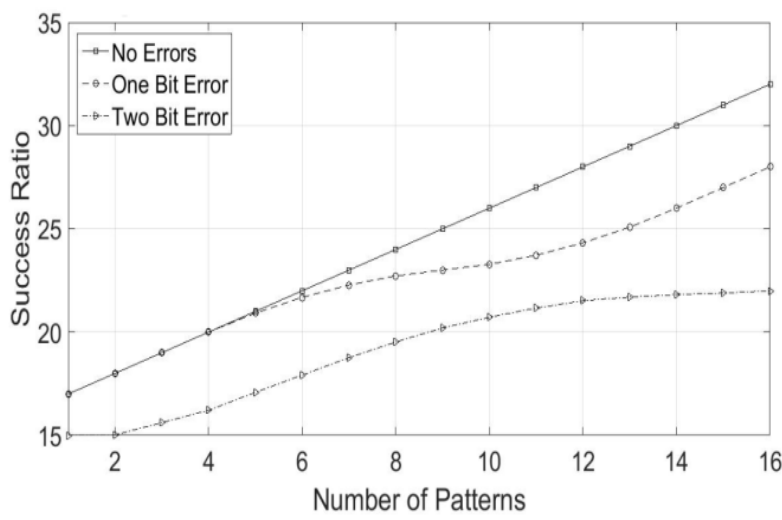

Fig. 9. Recognition Success Ratio for Bipolar Inputs

\section{CONCLUSION AND FUTURE WORK}

In this paper, models for the primary and secondary immune responses have been designed, trained and tested using a simple robotics simulation. During the primary artificial immune memory response, the designed model was able to respond properly to most environmental conditions. Oscillation took place at certain situations, because the knowledge in the memory model at that stage was still accumulating. On the other hand, during the secondary artificial immune memory response, even while having different levels of noise, the input patterns were recognized successfully with an acceptable recognition rates. In conclusion, the aforementioned results show that the use of idiotopic artificial immune networks and hetero-associative networks as models for the artificial immune memory is reliable and could produce acceptable recognition rates.

The designed memory models showed $100 \%$ recognition rates for the tested patterns. Even after adding noise to the input data, the overall memory model showed acceptable recognition rate of $87.5 \%$ for $25 \%$ noise, and $68.7 \%$ recognition rate for $50 \%$ noise. Based on the findings of this research, the designed memory system models are reliable and could be useful in similar applications utilizing the artificial immune memory learning capability.

The proposed research work commits itself to keep the design of both memory models as generic as possible, therefore, leaving the door open for diverse topics to be explored. For instance, for future work, to improve the recognition rates in the designed model, authors can modify the proposed models by designing a hybrid network model. In that regard, a backpropagation network could be used to produce an approximated response in case of noisy inputs. In that case, 
the seondary artificial immune memory response could be a combination of both the hetero-associative network as well as the back-propagation network responses. Thus, in future research, other hybrid models could be investigated, thereby further enhancing the recognition rate of the artificial immune system memory.

\section{REFERENCES}

[1] Dipankar Dasgupta, Luis F. Niño, Immunological Computation: Theory and Application. NewYork, NY : CRC Press, 2009.

[2] Ehud Lamm , Ron Unger, Biological Computation: Chapman and Hall/CRC Publishing, 2011

[3] D. Dasgupta, "Advances in Artificial Immune Systems," IEEE Computational Intelligence Magazine, Vol. 1, No. 4, pp. 40-49, 2006

[4] Lee, D., H. Jun and K. Sim., "Artificial Immune System for Realization of Co-Operative Strategies and Group Behavior in Collective Autonomous Mobile Robots," Proceedings of Fourth International Symposium on Artificial Life and Robotics, AAAI, Oita, Japan, pp. 232$235,1999$.

[5] Singh, S. P. N. and S. M. Thayer, "Immunology Directed Methods for Distributed Robotics: A novel, ImmunityBased Architecture for Robust Control \& Coordination," The Proceedings of SPIE: Mobile Robots XVI, Vol. 4573, November 2001.

[6] Purbasari, A., Iping, S.S., Santoso, O.S.; Mandala, R., "Designing Artificial Immune System Based on Clonal Selection: Using Agent-Based Modeling Approach," Modelling Symposium (AMS), 2013 7th Asia , vol., no., pp.11,15, 2013.

[7] Ying Tan, Guyue Mi; Yuanchun Zhu, Chao Deng, "Artificial Immune System Based Methods for Spam Filtering," Circuits and Systems (ISCAS), 2013 IEEE International Symposium on , vol., no., pp.2484,2488, 19-23 May 2013.

[8] Chung-Ming Ou, Ou, C. R., "Immune Memory with Associativity: Perspectives on Dynamical Systems," Evolutionary Computation (CEC), 2012 IEEE Congress on , vol., no., pp.1,6, 10-15 June 2012.

[9] Chung-Ming Ou, Ou, C.R., "Immune Network and Immune Memory Mechanism: Perspectives on Dynamical Systems," Technologies and Applications of
Artificial Intelligence (TAAI), 2011 International Conference on , vol., no., pp.313,318, 11-13 Nov. 2011.

[10] Alexandrino, J.L., Zanchettin, C.; Carvalho Filho, E., "Artificial Immune System with ART Memory Hibridization," Hybrid Intelligent Systems, 2007. HIS 2007. 7th International Conference on , vol., no., pp.59,64, 17-19 Sept. 2007.

[11] Yap, D.F.W., Koh, S. P.; Tiong, S.K., "Artificial Immune System Based on Hybrid and External Memory for Mathematical Function Optimization," Computers \& Informatics (ISCI), 2011 IEEE Symposium on , vol., no., pp.12,17, 20-23 March 2011.

[12] Meshref, H., "The Rod-Bearing Problem: A Cooperative Autonomous Robotics Application Based on Artificial Idiotopic Immune Networks," Systems, Man, and Cybernetics (SMC), 2013 IEEE International Conference on , vol., no., pp.1654,1659, 2013.

[13] N. K. Jerne., "Idiotopic Network and Other preconceived ideas," Immunological Rev., Vol. 79, pp. 5-24, 1984.

[14] N. K. Jerne., "The Generative Grammar of the Immune System," The EMBO Journal, 4(4): pp. 847-852, 1985.

[15] Leandro Nunes de Castro, Jonathan Timmis, Artificial Immune Systems: A New Computational Intelligence Approach: Springer, 2002

[16] Seral Şahan, Kemal Polat, Halife Kodaz, Salih Günes. "A New Hybrid Method Based on Fuzzy-Artificial Immune System and k-nn Algorithm for Breast Cancer Diagnosis," Computers in Biology and Medicine Volume 37, Issue 3, pp. 415-423, 2007.

[17] Hongwei Mo, Handbook of Research on Artificial Immune Systems and Natural Computing: Applying Complex Adaptive Technologies: Igi Global Publisher, 2009.

[18] Rob J. de Boer. Theoretical Biology \& Bioinformatics: Utrecht University Press, 2013.

[19] Simon Hykins. Neural Networks: A Comprehensive Foundation, Prentice Hall, 1999.

[20] Simon O. Haykin, Neural Networks and Learning Machines. Upper Saddle River, New Jersy: Pearson Education, 2009. 